\title{
Hamilton-Jacobi Approach for First Order Actions and Theories with Higher Derivatives
}

\author{
M. C. $\operatorname{Bertin}^{(a)}$ 1, B. M. Pimentel ${ }^{(a)} \sqrt[2]{2}$, P. J. Pompeia $(a, b) \sqrt[3]{3}$ \\ (a) Instituto de Física Teórica - Universidade Estadual Paulista, \\ Rua Pamplona 145, 01405-900, São Paulo, SP, Brazil. \\ Telephone +55 11 3177-9029, FAX +55 11 3177-9080 \\ (b) Comando-Geral de Tecnologia Aeroespacial - Instituto de Fomento e \\ Coordenação Industrial - Divisão de Confiabilidade Metrológica Aeroespacial \\ Praça Mal. Eduardo Gomes, 50, 12228-901, São José dos Campos, SP, Brazil.

\begin{abstract}
In this work we analyze systems described by Lagrangians with higher order derivatives in the context of the Hamilton-Jacobi formalism for first order actions. Two different approaches are studied here: the first one is analogous to the description of theories with higher derivatives in the hamiltonian formalism according to [11, 12]; the second treats the case where degenerate coordinate are present, in an analogy to reference [13]. Several examples are analyzed where a comparison between both approaches is made.
\end{abstract}

PACS Nos: 11.10.Ef, 45.20.-d

Keywords: Hamilton-Jacobi formalism, singular systems, first order actions, higher order derivatives.

\section{Introduction}

The interest of physicists by systems described by Lagrangians with derivatives higher than one is not recent. Since 1850, when Ostrogradski developed the first work concerning the hamiltonian formalism for systems with higher derivatives [1], systems of this type have been used in many relevant problems of Physics. As examples one can cite the works of Podolsky 22 and Bopp [3, who independently proposed generalizations of Electrodynamics containing second order derivatives, and the works of Green 4, who proposed a generalized meson-field theory. Many other applications can be found in literature [5, 6, 7, 8, where systems with higher derivatives have been successfully used. When dealing with

\footnotetext{
${ }^{1}$ mcbertin@ift.unesp.br

2 pimentel@ift.unesp.br

3 pompeia@ift.unesp.br
} 
systems of this type, special attempts must be made when the Lagrangian is singular.

As it is well known, the usual approach to deal with singular systems was developed by Dirac in the early 1950‘s 9, 10, and its application to system with higher derivatives was made in the 1980's [11, 12, which we refer henceforth as the standard approach. Very recently, a new development for systems with higher derivatives and degenerate coordinates, i.e. coordinates whose derivatives are not present in the theory (refered from now on as the degenerate coordinates approach), was made by Gitman and Tyutin [13] and a new definition of singularity of a theory was proposed. According to the authors, this new definition would be strictly correlated to the gauge character of the theory. One interesting feature of both these developments lies on the fact that a Lagrangian linear in the velocities can be written down.

Although widely accepted, Dirac formalism did not avoid the appearance of other approaches, which always provide new points of view for the same problems. One of them is the Hamilton-Jacobi (HJ) formalism, based on the Carathéodory Equivalent Lagrangian method [14, whose approach to singular systems was developed by Güler [15, and since several applications and extra developments have been made [16, 17, 18, 19, 20, 21], including the study of systems with second and higher order derivatives [22, 24]. An important application in the context of this work was recently made in reference 25, where systems described by first order actions, i.e. Lagrangians linear in the velocities, were studied via HJ formalism. In 25 it was also shown how generalized brackets can be constructed and how it is related to the existence of a symplectic structure in this formalism.

In this work we intend to analyze how systems with higher derivatives are described in the HJ first order context. Considering that two different approaches, the standard one and that with degenerate coordinates, were made for systems with higher derivatives in the hamiltonian description, we intend to compare how both of them can be described in the HJ formalism and see what differences can be pointed out. For this in the next section we will make a review of the HJ first order actions. In sequence we will apply this structure for systems with higher order derivatives in analogy to the standard approach (SA). Then the same will be made in the degenerate coordinates context (DC). Several examples will be analyzed and the differences will be pointed out. At last some remarks will be made.

\section{First Order Actions in HJ Formalism}

Let us consider the following Lagrangian

$$
L\left(z_{B}, \dot{z}_{B}\right)=\dot{z}_{A} K^{A}\left(z_{B}\right)-V\left(z_{B}\right), \quad A, B=1, \ldots, N .
$$


One then identifies the constraints

$$
\begin{aligned}
\phi^{A} & \equiv p^{A}-K^{A}(z)=0, \\
\phi^{0} & \equiv p^{0}+V(z)=0 .
\end{aligned}
$$

According to reference [25], to verify the integrability conditions of this system, one must analyze the matrix

$$
M^{A B} \equiv\left\{\phi^{A}, \phi^{B}\right\}=\frac{\partial K^{B}}{\partial z_{A}}-\frac{\partial K^{A}}{\partial z_{B}} .
$$

If $\left\{\phi^{A}, \phi^{0}\right\}=0, M^{A B}=0$, then system is integrable. When this is not the case, relations between the $z_{B}$ are stablished, leading one to define generalized brackets. When the matrix $M^{A B}$ has rank $P=N-R$, then a submatrix $P \times P$ exists such that

$$
\operatorname{det}\left(M^{\dot{a} \dot{b}}\right) \neq 0, \quad \dot{a}, \dot{b}=1, \ldots, P,
$$

which means that $M_{\dot{a} \dot{b}}^{-1}$ also exists. The identification of this submatrix separate $z_{B}$ in two sets

$$
z_{B} \rightarrow\left\{\begin{array}{cc}
t_{\dot{b}}, & \dot{b}=1, \ldots, P \\
t_{\beta}, & \beta=1, \ldots, R
\end{array}\right.
$$

where $t_{\dot{b}}$ are the true dynamical variables and $t_{\beta}$ play the role of parameters in the theory. When this separation is done, some integrability conditions must be satisfied:

$$
\left\{\phi^{\alpha}, \phi^{\beta}\right\}=\left\{\phi^{\alpha}, \phi^{\dot{b}}\right\} M_{\dot{b} \dot{a}}^{-1}\left\{\phi^{\dot{a}}, \phi^{\beta}\right\} .
$$

Finally, generalized brackets can be constructed

$$
\{F, G\}_{*} \equiv\{F, G\}-\left\{F, \phi^{\dot{b}}\right\} M_{\dot{b} \dot{a}}^{-1}\left\{\phi^{\dot{a}}, G\right\},
$$

such that the differential of any function $E=E(z)$ becomes

$$
d E=\left\{E, \phi^{\beta^{\prime}}\right\}_{*} d t_{\beta^{\prime}}
$$

Hence the equations of motion can be obtained by setting $E=t_{\dot{b}}$.

\section{Theories with Higher Derivatives - Standard Approach}

In order to apply the structure of previous section to theories with higher derivatives one can employ the development of references [11, 12, which is described below. Let the system of interest be decribed by a Lagrangian

$$
L=L\left(q^{a}, q^{\left(l_{a}\right)}, t\right)
$$


where

$$
q^{a\left(l_{a}\right)} \equiv \frac{d^{l_{a}}}{d t^{l_{a}}} q^{a}, \quad\left(a=1, \ldots, n ; l_{a}=1, \ldots, N_{a}\right) .
$$

This theory is constructed in a configuration space with $n$ coordinates $q^{a}$. Instead of studying the theory in this space, let one consider a larger space with coordinates $x_{s}^{a}, v^{a}$ such that

$$
\left\{\begin{array}{l}
x_{s}^{a} \equiv q^{a(s-1)}, \quad s=1, \ldots, N_{a},\left(q^{a(0)} \equiv q^{a}\right) \\
v^{a} \equiv q^{a\left(N_{a}\right)}
\end{array}\right.
$$

With these definitions it can be stablished the following relations between some coordinates and some of their time derivatives

$$
\left\{\begin{array}{c}
x_{s+1}^{a}=\dot{x}_{s}^{a} \Rightarrow \dot{x}_{s}^{a}-x_{s+1}^{a}=0, \quad s=1, \ldots, N_{a}-1, \\
v^{a}=\dot{x}_{N_{a}}^{a} \Rightarrow \dot{x}_{N_{a}}^{a}-v^{a}=0 .
\end{array}\right.
$$

In this enlarged space the system can be described by the following Lagrangian

$$
L^{v} \equiv L\left(q^{a(s-1)}=x_{s}^{a}, q^{a\left(N_{a}\right)}=v^{a}, t\right)=L^{v}\left(x_{s}^{a}, v^{a}, t\right) .
$$

Since the relations (8) must be satisfied, one can use Lagrange multipliers to incorporate them in the theory:

$$
\begin{aligned}
\bar{L}^{v} & =\bar{L}^{v}\left(x_{s}^{a}, v^{a}, t ; \pi_{a}^{s}\right) \equiv L^{v}\left(x_{s}^{a}, v^{a}, t\right)+ \\
& +\sum_{a} \sum_{s=1}^{N_{a}-1} \pi_{a}^{s}\left(\dot{x}_{s}^{a}-x_{s+1}^{a}\right)+\sum_{a} \pi_{a}^{N_{a}}\left(\dot{x}_{N_{a}}^{a}-v^{a}\right) .
\end{aligned}
$$

It must be noticed that, with these multipliers, the theory is now constructed in larger space with coordinates $x_{s}^{a}, v^{a}, \pi_{a}^{s} . \bar{L}^{v}$ can be rewritten as a first order Lagrangian

$$
\begin{aligned}
\bar{L}^{v} & =\sum_{a} \sum_{s=1}^{N_{a}-1} \pi_{a}^{s} \dot{x}_{s}^{a}+\sum_{a} \pi_{a}^{N_{a}} \dot{x}_{N_{a}}^{a}-H^{v}\left(x_{s}^{a}, v^{a}, t ; \pi_{a}^{s}\right), \\
H^{v}\left(x_{s}^{a}, v^{a}, t ; \pi_{a}^{s}\right) & \equiv \sum_{a} \sum_{s=1}^{N_{a}-1} \pi_{a}^{s} x_{s+1}^{a}+\sum \pi_{a}^{N_{a}} v^{a}-L^{v}\left(x_{s}^{a}, v^{a}, t\right) .
\end{aligned}
$$

Now the procedure of the previous section can be applied

\subsection{HJ first order approach}

In (10) the functions $K^{A}$ and the constraints can be identified as

$$
\begin{gathered}
\left\{\begin{array}{c}
K_{a}^{v}=0 \\
K_{a}^{x_{s}}=\pi_{a}^{s} \\
K_{\pi_{s}}^{a}=0
\end{array}\right. \\
\phi^{t}=p^{0}+H^{v}=0 \\
\phi_{\pi_{s}}^{a}=p_{\pi_{s}}^{a}=0 \\
\phi_{a}^{x_{s}}=p_{a}^{x_{s}}-\pi_{a}^{s}=0
\end{gathered}
$$


which allow one to construct the matrix $M^{A B}$ :

$$
\begin{aligned}
& M^{A B}=\frac{\partial K^{B}}{\partial z_{A}}-\frac{\partial K^{A}}{\partial z_{B}}, \\
& \left(M^{A B}\right)=\left(\begin{array}{ccc}
0 & \frac{\partial K_{b}^{x_{s}}}{\partial v^{a}}-\frac{\partial K_{a}^{v}}{\partial x_{s}^{b}} & \frac{\partial K_{\pi_{s}}^{c}}{\partial v^{a}}-\frac{\partial K_{a}^{v}}{\partial \pi_{c}^{c^{\prime}}} \\
\frac{\partial K_{a}^{v}}{\partial x_{s}^{b}}-\frac{\partial K_{b}^{x_{s}}}{\partial v^{a}} & 0 & \frac{\partial K_{\pi_{s^{\prime}}}^{c}}{\partial x_{s}^{b}}-\frac{\partial K_{b}^{x}}{\partial \pi_{c}^{s^{\prime}}} \\
\frac{\partial K_{s}^{v}}{\partial \pi_{c}^{s}}-\frac{\partial K_{\pi_{s}}^{c}}{\partial v^{a}} & \frac{\partial K_{b}^{x_{s}}}{\partial \pi_{c}^{s^{\prime}}}-\frac{\partial K_{\pi_{s} s^{\prime}}^{c}}{\partial x_{s}^{s}} & 0
\end{array}\right)= \\
& =\left(\begin{array}{ccc}
0 & 0 & 0 \\
0 & 0 & -\delta_{s^{\prime}}^{s} \delta_{b}^{c} \\
0 & \delta_{s^{\prime}}^{s} \delta_{b}^{c} & 0
\end{array}\right) .
\end{aligned}
$$

This matrix is singular, but there is an inversible submatrix

$$
\begin{aligned}
& \left(M^{\dot{c} \dot{b}}\right)=\left(\begin{array}{cc}
0 & -\delta_{s^{\prime}}^{s} \delta_{b}^{c} \\
\delta_{s^{\prime}}^{s} \delta_{b}^{c} & 0
\end{array}\right), \\
& \left(M_{\dot{a} \dot{c}}^{-1}\right)=\left(\begin{array}{cc}
0 & \delta_{s^{\prime}}^{s} \delta_{c}^{a} \\
-\delta_{s^{\prime}}^{s} \delta_{c}^{a} & 0
\end{array}\right),
\end{aligned}
$$

showing that the variables can be separated in two sets:

$$
\begin{aligned}
t_{\dot{a}} & =\left\{x_{s}^{a} ; \pi_{a}^{s}\right\}, \\
t_{\beta} & =\left\{v^{a}\right\} .
\end{aligned}
$$

With this separation one must stablish the integrability conditions

$\left\{\phi^{\beta}, \phi^{t}\right\}=\left\{\phi^{\beta}, \phi^{\dot{a}}\right\} M_{\dot{a} \dot{b}}^{-1}\left\{\phi^{\dot{b}}, \phi^{t}\right\}, \quad \phi^{\dot{a}}=\left\{\phi_{a}^{x_{s}} ; \phi_{\pi_{s}}^{a}\right\}, \phi^{\beta}=\left\{\phi_{a}^{v}\right\}$.

Since $\left\{\phi^{\dot{b}}, \phi_{a}^{v}\right\}=0$, as it can be verified in the matrix $M^{A B}$, it follows

$$
\begin{aligned}
\left\{\phi^{\beta}, \phi^{t}\right\} & =0 \Rightarrow\left\{\phi_{b}^{v}, \phi^{t}\right\}=-\frac{\partial \phi^{t}}{\partial v^{b}}=\frac{\partial H^{v}}{\partial v^{b}}=0 \Rightarrow \\
& \Rightarrow \pi_{a}^{N_{a}}-\frac{\partial L^{v}}{\partial v^{a}}=0 .
\end{aligned}
$$

Notice that these conditions stablish some of the Lagrange multipliers.

The next step is to stablish the Generalized Brackets, which allows one to evaluate the total differential of any function $E=E\left(x_{s}^{a}, v^{a}, \pi_{a}^{s}\right)$. For this it is necessary to know the following quantities $\left\{\phi^{\dot{a}}, \phi^{\beta}\right\}$ and $\left\{\phi^{\dot{a}}, \phi^{t}\right\}$. As mentioned before, the former are null and only the last must be specified. First it mus be noticed that, for any function $E=E\left(x_{s}^{a}, v^{a}, \pi_{a}^{s}\right)$, one has:

$$
\begin{gathered}
\left\{\phi_{a}^{x_{s}}, E\right\}=-\frac{\partial E}{\partial x_{s}^{a}}, \\
\left\{\phi_{\pi_{s}}^{a}, E\right\}=-\frac{\partial E}{\partial \pi_{a}^{s}} .
\end{gathered}
$$


For $E=\phi^{t}$ it follows

$$
\left\{\phi^{\dot{a}}, \phi^{t}\right\} \rightarrow\left\{\begin{array}{l}
\left\{\phi_{a}^{x_{s}}, \phi^{t}\right\}=-\frac{\partial \phi^{t}}{\partial x_{s}^{a a}} \\
\left\{\phi_{\pi_{s}}^{a}, \phi^{t}\right\}=-\frac{\partial \phi^{t}}{\partial \pi_{a}^{s}}
\end{array} .\right.
$$

This way

$$
\begin{gathered}
d E=\left\{E, \phi^{\beta^{\prime}}\right\}_{*} d t_{\beta^{\prime}}=\left\{E, \phi^{t}\right\}_{*} d t+\left\{E, \phi_{a}^{v}\right\}_{*} d v^{a}, \\
d E=\left\{E, \phi^{t}\right\} d t-\left\{E, \phi^{\dot{a}}\right\} M_{\dot{a} \dot{b}}^{-1}\left\{\phi^{\dot{b}}, \phi^{t}\right\} d t+ \\
+\left\{E, \phi_{a}^{v}\right\} d v^{a}-\left\{E, \phi^{\dot{a}}\right\} M_{\dot{a} \dot{b}}^{-1}\left\{\phi^{\dot{b}}, \phi_{a}^{v}\right\} d v^{a} \\
d E=\frac{\partial E}{\partial t} d t+\frac{\partial E}{\partial v^{a}} d v^{a}+\left[\frac{\partial E}{\partial x_{s}^{c}} \frac{\partial \phi^{t}}{\partial \pi_{c}^{s}}-\frac{\partial \phi^{t}}{\partial x_{s}^{c}} \frac{\partial E}{\partial \pi_{c}^{s}}\right] d t .
\end{gathered}
$$

Now the equations of motion can be obtained

$$
\begin{gathered}
d t_{\dot{a}}=\frac{\partial t_{\dot{a}}}{\partial t} d t+\frac{\partial t_{\dot{a}}}{\partial v^{a}} d v^{a}+\left[\frac{\partial t_{\dot{a}}}{\partial x_{s}^{c}} \frac{\partial \phi^{t}}{\partial \pi_{c}^{s}}-\frac{\partial \phi^{t}}{\partial x_{s}^{c}} \frac{\partial t_{\dot{a}}}{\partial \pi_{c}^{s}}\right] d t \Rightarrow d x_{s}^{a}=\frac{\partial H^{v}}{\partial \pi_{a}^{s}} d t \\
d \pi_{a}^{s}=-\frac{\partial \phi^{t}}{\partial x_{s}^{a}} d t=-\frac{\partial H^{v}}{\partial x_{s}^{a}} d t \Rightarrow\left\{\begin{array}{l}
d \pi_{a}^{s}=-\left(\pi_{a}^{s-1}-\frac{\partial L^{v}}{\partial x_{s}^{a}}\right) d t, \quad s=2, \ldots, N_{a}, \\
d \pi_{a}^{1}=\frac{\partial L^{v}}{\partial x_{1}^{a}} d t .
\end{array}\right.
\end{gathered}
$$

Equations (13) are consistent with (8), while from (14) it can be seen that the other Lagrange multipliers are determined:

$$
\pi_{a}^{s-1}=-\dot{\pi}_{a}^{s}+\frac{\partial L^{v}}{\partial x_{s}^{a}}, \quad s=2, \ldots, N_{a} .
$$

\section{Degenerate Coordinates Approach}

Also with the intention to study the HJ approach for systems with degenerate coordinates, one can start following the proposal made by Gitman and Tyutin in reference [13, in which a first order Lagrangian is proposed.

Let one consider a Lagrangian

$$
L=L\left(q^{a}, q^{\left(l_{a}\right)}, t\right)
$$

where

$$
q^{\left(l_{a}\right)} \equiv \frac{d^{l_{a}}}{d t^{l_{a}}} q^{a}, \quad\left(a=1, \ldots, n ; l_{a}=1, \ldots, N_{a}\right) .
$$

The coordinates $q^{a}$ will be separated in two sets, $q^{\bar{a}}$ and $q^{\tilde{a}}$, where $q^{\bar{a}}$ are coordinates whose derivatives are not present in the Lagrangian, i.e. degenerate 
coordinates, and $q^{\tilde{a}}$ are those whose derivatives of order $N_{\tilde{a}}$ are manifest in $L$, i.e.

$$
a=(\bar{a}, \tilde{a}), \quad N_{\bar{a}}=0 ; N_{\tilde{a}} \geq 1 .
$$

One then defines new coordinates

$$
\begin{aligned}
v^{\bar{a}} & \equiv q^{\bar{a}}, \\
x_{1}^{\tilde{a}} & \equiv q^{\tilde{a}} \\
x_{s}^{\tilde{a}} & \equiv q^{\tilde{a}(s-1)}, \quad s=2, \ldots, N_{\tilde{a}}, \\
v^{\tilde{a}} & \equiv q^{\tilde{a}\left(N_{\tilde{a}}\right)},
\end{aligned}
$$

from where the following relations are identified:

$$
\begin{aligned}
x_{s+1}^{\tilde{a}} & =\dot{x}_{s}^{\tilde{a}} \Rightarrow \dot{x}_{s}^{\tilde{a}}-x_{s+1}^{\tilde{a}}=0, \quad s=1, \ldots, N_{\tilde{a}}-1, \\
v^{\tilde{a}} & =\dot{x}_{N_{\tilde{a}}^{\tilde{a}}}^{\tilde{a}} \Rightarrow \dot{x}_{N_{\tilde{a}}}^{\tilde{a}}-v^{\tilde{a}}=0 .
\end{aligned}
$$

With these new coordinates $L$ becomes

$$
L^{v} \equiv L\left(q^{\bar{a}}=v^{\bar{a}}, q^{\tilde{a}(s-1)}=x_{s}^{\tilde{a}}, q^{\tilde{a}\left(N_{\tilde{a}}\right)}=v^{\tilde{a}}, t\right)=L^{v}\left(x_{s}^{\tilde{a}}, v^{a}, t\right) .
$$

From this expression it is possible to define a new Lagrangian, $\bar{L}^{v}$, in an extended space with coordinates $\left\{x_{s}^{\tilde{a}}, v^{a}, \pi_{\tilde{a}}^{s}\left(s=1, \ldots, N_{\tilde{a}}\right)\right\}$, which differs from $L^{v}$ by the presence of Lagrange multipliers, $\pi_{\tilde{a}}^{s}$ :

$$
\begin{aligned}
\bar{L}^{v} & =\bar{L}^{v}\left(x_{s}^{\tilde{a}}, v^{a}, t ; \pi_{\tilde{a}}^{s}\right) \equiv L^{v}\left(x_{s}^{\tilde{a}}, v^{a}, t\right)+ \\
& +\sum_{\tilde{a}} \sum_{s=1}^{N_{\tilde{a}}-1} \pi_{\tilde{a}}^{s}\left(\dot{x}_{s}^{\tilde{a}}-x_{s+1}^{\tilde{a}}\right)+\sum \pi_{\tilde{a}}^{N_{\tilde{a}}}\left(\dot{x}_{N_{\tilde{a}}^{\tilde{a}}}^{\tilde{a}}-v^{\tilde{a}}\right) .
\end{aligned}
$$

$\bar{L}^{v}$ is a first order Lagrangian, what becomes clearer when it is rewritten as $L=\dot{z}_{A} K^{A}-V\left(z_{A}\right)$, where $z_{A}=\left(x_{s}^{\tilde{a}}, v^{a} ; \pi_{\tilde{a}}^{s}\right), s=1, \ldots, N_{\tilde{a}}$ :

$$
\begin{aligned}
\bar{L}^{v} & =\sum_{\tilde{a}} \sum_{s=1}^{N_{\tilde{a}}-1} \pi_{\tilde{a}}^{s} \dot{x}_{s}^{\tilde{a}}+\sum \pi_{\tilde{a}}^{N_{\tilde{a}}} \dot{x}_{N_{\tilde{a}}}^{\tilde{a}}-H^{v}\left(x_{s}^{\tilde{a}}, v^{a}, t ; \pi_{\tilde{a}}^{s}\right), \\
H^{v}\left(x_{s}^{\tilde{a}}, v^{a}, t ; \pi_{\tilde{a}}^{s}\right) & \equiv \sum_{\tilde{a}} \sum_{s=1}^{N_{\tilde{a}}-1} \pi_{\tilde{a}}^{s} x_{s+1}^{\tilde{a}}+\sum \pi_{\tilde{a}}^{N_{\tilde{a}}} v^{\tilde{a}}-L^{v}\left(x_{s}^{\tilde{a}}, v^{a}, t\right) .
\end{aligned}
$$

The procedure of reference 25 now can be applied.

It is important to notice that the main difference between the standard approach and this one lies on the definition of the velocities of the degenerate coordinates as the coordinates themselves in this last development. When no degenerate coordinates exist, both approaches coincide; when they have presence in $L$, the definitions of the velocities of degenerate coordinates are different and one can verify that the space of the first approach is larger than the space of the second. 


\subsection{HJ first order approach}

The first step is to identify the functions $K^{A}$ and the constraints. It follows

$$
\begin{gathered}
\left\{\begin{array}{c}
K_{a}^{v}=0 \\
K_{\tilde{a}_{s}}^{x_{s}}=\pi_{\tilde{a}}^{s} \\
K_{\pi_{s}}^{\tilde{a}}=0
\end{array}\right. \\
\phi^{t}=p^{0}+H^{v}=0 . \\
\phi_{\tilde{a}}^{x_{s}}=p_{a}^{v}=p_{a}^{v}=0 \\
\phi_{\pi_{s}}^{\tilde{a}}=p_{\pi_{s}}^{\tilde{a}}=0
\end{gathered}
$$

The next step is to compute the matrix $M^{A B}$ :

$$
\begin{aligned}
& M^{A B}=\frac{\partial K^{B}}{\partial z_{A}}-\frac{\partial K^{A}}{\partial z_{B}}, \\
& \left(M^{A B}\right)=\left(\begin{array}{ccc}
0 & \frac{\partial K_{\tilde{b}}^{x_{s}}}{\partial v^{a}}-\frac{\partial K_{a}^{v}}{\partial x_{s}^{b}} & \frac{\partial K_{\pi_{s}}^{\tilde{a}}}{\partial v^{a}}-\frac{\partial K_{a}^{v}}{\partial \pi_{\tilde{a}}^{s}} \\
\frac{\partial K_{a}^{v}}{\partial x_{s}^{b}}-\frac{\partial K_{\tilde{b}}^{x_{s}}}{\partial v^{a}} & 0 & \frac{\partial K_{\pi_{s}^{\prime}}^{\tilde{a}}}{\partial x_{s}^{b}}-\frac{\partial K_{\tilde{b}}^{x_{s}}}{\partial \pi_{\tilde{a}}^{s^{\prime}}} \\
\frac{\partial K_{a}^{v}}{\partial \pi_{\tilde{a}}^{s}}-\frac{\partial K_{\pi_{s}}^{\tilde{a}}}{\partial v^{a}} & \frac{\partial K_{\tilde{b}}^{x_{s}}}{\partial \pi_{\tilde{a}}^{s^{\prime}}}-\frac{\partial K_{\pi_{s^{\prime}}}^{\tilde{a}}}{\partial x_{s}^{b}} & 0
\end{array}\right)= \\
& =\left(\begin{array}{ccc}
0 & 0 & 0 \\
0 & 0 & -\delta_{s^{\prime}}^{s} \delta_{\tilde{b}}^{\tilde{a}} \\
0 & \delta_{s^{\prime}}^{s} \delta_{\tilde{b}}^{\tilde{a}} & 0
\end{array}\right) .
\end{aligned}
$$

This matrix is singular with an inversible submatrix $M^{\dot{a} \dot{b}}$ :

$$
\begin{aligned}
& \left(M^{\dot{a} \dot{b}}\right)=\left(\begin{array}{cc}
0 & -\delta_{s^{\prime}}^{s} \delta_{\tilde{b}}^{\tilde{a}} \\
\delta_{s^{\prime}}^{s} \delta_{\tilde{b}}^{\tilde{a}} & 0
\end{array}\right), \\
& \left(M_{\dot{a} \dot{b}}^{-1}\right)=\left(\begin{array}{cc}
0 & \delta_{s^{\prime}}^{s} \delta_{\tilde{b}}^{\tilde{a}} \\
-\delta_{s^{\prime}}^{s} \delta_{\tilde{b}}^{\tilde{a}} & 0
\end{array}\right),
\end{aligned}
$$

which shows that the coordinates are separated in two sets:

$$
\begin{aligned}
t_{\dot{a}} & =\left\{x_{s}^{\tilde{a}} ; \pi_{\tilde{a}}^{s}\right\}, \\
t_{\beta} & =\left\{v^{a}\right\} .
\end{aligned}
$$

The integrability conditions must be determined:

$$
\left\{\phi^{\beta}, \phi^{t}\right\}=\left\{\phi^{\beta}, \phi^{\dot{a}}\right\} M_{\dot{a} \dot{b}}^{-1}\left\{\phi^{\dot{b}}, \phi^{t}\right\} .
$$

Since $\left\{\phi^{\dot{b}}, \phi_{a}^{v}\right\}=0$, it follows

$$
\left\{\phi^{\beta}, \phi^{t}\right\}=0 \Rightarrow\left\{\phi_{b}^{v}, \phi^{t}\right\}=\frac{\partial H^{v}}{\partial v^{b}}=0 \Rightarrow\left\{\begin{array}{c}
\pi_{\tilde{a}}^{N_{\tilde{a}}}-\frac{\partial L^{v}}{\partial v^{a}}=0 \\
\frac{\partial L^{v}}{\partial v^{\bar{a}}}=0
\end{array} .\right.
$$

With these conditions some of the Lagrange multipliers are fixed. 
Now the Generalized Brackets can be constructed, and for this, $\left\{\phi^{\dot{a}}, \phi^{t}\right\}$ must be determined:

$$
\left\{\phi^{\dot{a}}, \phi^{t}\right\} \rightarrow\left\{\begin{array}{l}
\left\{\phi_{\tilde{a}}^{x_{s}}, \phi^{t}\right\}=-\frac{\partial \phi^{t}}{\partial x_{s}^{a}} \\
\left\{\phi_{\pi_{s}}^{\tilde{a}}, \phi^{t}\right\}=-\frac{\partial \phi^{t}}{\partial \pi_{\tilde{a}}^{s}}
\end{array} .\right.
$$

The total differential of $E=E\left(x_{s}^{\tilde{a}}, v^{a}, \pi_{\tilde{a}}^{s}\right)$ is

$$
d E=\left\{E, \phi^{\beta^{\prime}}\right\}_{*} d t_{\beta^{\prime}}=\left\{E, \phi^{t}\right\}_{*} d t+\left\{E, \phi_{a}^{v}\right\}_{*} d v^{a}
$$

Since for any pair of functions $E=E\left(x_{s}^{\tilde{a}}, v^{a}, \pi_{\tilde{a}}^{s}\right)$ and $F=F\left(x_{s}^{\tilde{a}}, v^{a}, \pi_{\tilde{a}}^{s}\right)$ one has $\{E, F\}=0$, it follows, considering $H^{v}=H^{v}\left(x_{s}^{\tilde{a}}, v^{a}, t ; \pi_{\tilde{a}}^{s}\right)$, that

$$
d E=\frac{\partial E}{\partial t} d t+\frac{\partial E}{\partial v^{a}} d v^{a}+\left[\frac{\partial E}{\partial x_{s}^{\tilde{c}}} \frac{\partial \phi^{t}}{\partial \pi_{\tilde{c}}^{s}}-\frac{\partial \phi^{t}}{\partial x_{s}^{\tilde{c}}} \frac{\partial E}{\partial \pi_{\tilde{c}}^{s}}\right] d t .
$$

At last the equations of motion are obtained:

$$
\begin{gathered}
d t_{\dot{a}}=\frac{\partial t_{\dot{a}}}{\partial t} d t+\frac{\partial t_{\dot{a}}}{\partial v^{a}} d v^{a}+\left[\frac{\partial t_{\dot{a}}}{\partial x_{s}^{\tilde{c}}} \frac{\partial \phi^{t}}{\partial \pi_{\tilde{c}}^{s}}-\frac{\partial \phi^{t}}{\partial x_{s}^{\tilde{c}}} \frac{\partial t_{\dot{a}}}{\partial \pi_{\tilde{c}}^{s}}\right] d t \\
d x_{s}^{\tilde{a}}=\frac{\partial \phi^{t}}{\partial \pi_{\tilde{a}}^{s}} d t=\frac{\partial H^{v}}{\partial \pi_{\tilde{a}}^{s}} d t \\
d \pi_{\tilde{a}}^{s}=-\frac{\partial \phi^{t}}{\partial x_{s}^{\tilde{a}}} d t=-\frac{\partial H^{v}}{\partial x_{s}^{\tilde{a}}} d t \Rightarrow\left\{\begin{array}{l}
d \pi_{\tilde{a}}^{S}=-\left(\pi_{\tilde{a}}^{s-1}-\frac{\partial L^{v}}{\partial x_{s}^{\tilde{a}}}\right) d t, \quad s=2, \ldots, N_{\tilde{a}}, \\
d \pi_{\tilde{a}}^{1}=\frac{\partial L^{\delta}}{\partial x_{1}^{\tilde{a}}} d t .
\end{array}\right.
\end{gathered}
$$

One observes that the remaining Lagrange multipliers (those not determined by integrability conditions) are now fixed by the equations of motion:

$$
\pi_{\tilde{a}}^{s-1}=-\dot{\pi}_{\tilde{a}}^{s}+\frac{\partial L^{v}}{\partial x_{s}^{\tilde{a}}}, \quad s=2, \ldots, N_{\tilde{a}} .
$$

\section{$5 \quad$ Examples}

\subsection{Podolsky Electrodynamics}

As it can be observed, when no degenerate coordinates exist, both approaches coincide. As a first example it will be considered the case of Podolsky Electrodynamics, which is a theory with second derivatives and no degenerate coordinates. The intention is to show that the results obtained with the first order approach is consistent with the results obtained by the standard HJ approach for systems with higher order derivatives [22] and those obtained with Dirac's approach [23]. Podolsky Electrodynamics Lagrangian is given by:

$$
L=-\frac{1}{4} F_{\mu \nu} F^{\mu \nu}+a^{2} \partial_{\rho} F^{\mu \rho} \partial_{\sigma} F_{\mu}^{\cdot \sigma},
$$


where $F_{\mu \nu}=\partial_{\mu} A_{\nu}-\partial_{\nu} A_{\mu} . L$ can be rewritten as

$$
\begin{aligned}
L & =-\frac{1}{4}\left[2 \eta^{00} \eta^{i j}\left(\partial_{0} A_{i}-\partial_{i} A_{0}\right)\left(\partial_{0} A_{j}-\partial_{j} A_{0}\right)+F_{i j} F^{i j}\right]+ \\
& +a^{2}\left[\eta^{i j} \eta^{00} \eta^{00}\left(\partial_{j} \partial_{0} A_{0}-\partial_{0} \partial_{0} A_{j}\right)\left(\partial_{i} \partial_{0} A_{0}-\partial_{0} \partial_{0} A_{i}\right)+\right. \\
& +\eta^{i k} \eta^{00} \eta^{j m}\left(\partial_{i} \partial_{0} A_{k}-\partial_{i} \partial_{k} A_{0}\right)\left(\partial_{j} \partial_{0} A_{m}-\partial_{j} \partial_{m} A_{0}\right)+ \\
& +2 \eta^{i k} \eta^{00} \eta^{j m}\left(\partial_{k} \partial_{0} A_{0}-\partial_{0} \partial_{0} A_{k}\right) \partial_{j} F_{i m}+ \\
& \left.\left.+\eta^{j l} \eta^{i m} \eta^{k n} \partial_{i} F_{l m} \partial_{k} F_{j n}\right)\right],
\end{aligned}
$$

which shows explicitly the time derivatives of $A_{\nu}$. It is immediate to identify that $L=L\left(A_{0}, A_{i}, \partial_{0} A_{0}, \partial_{0} A_{i}, \partial_{0}^{2} A_{i}\right)$, i.e $. N_{A_{0}}=1, N_{A_{i}}=2$. Introducing new variables

$$
\begin{aligned}
x_{0}^{(1)} & \equiv A_{0}, \quad v_{0} \equiv \partial_{0} A_{0}=\partial_{0} x_{0}^{(1)}, \\
x_{i}^{(1)} & \equiv A_{i}, \\
x_{i}^{(2)} & \equiv \partial_{0} A_{i}=\partial_{0} x_{i}^{(1)}, \quad v_{i} \equiv \partial_{0} \partial_{0} A_{i}=\partial_{0} x_{i}^{(2)},
\end{aligned}
$$

and defining

$$
F_{i j}^{x_{1}} \equiv \partial_{i} x_{j}^{(1)}-\partial_{j} x_{i}^{(1)}
$$

one obtains

$$
\begin{aligned}
L^{v} & =\left.L\right|_{A=x, \partial A=x, \partial^{2} A=v}=-\frac{1}{4}\left[2 \eta^{00} \eta^{i j}\left(x_{i}^{(2)}-\partial_{i} x_{0}^{(1)}\right)\left(x_{j}^{(2)}-\partial_{j} x_{0}^{(1)}\right)+F_{i j}^{x_{1}} F_{x_{1}}^{i j}\right]+ \\
& +a^{2}\left[\eta^{i j} \eta^{00} \eta^{00}\left(\partial_{j} v_{0}-v_{j}\right)\left(\partial_{i} v_{0}-v_{i}\right)+\right. \\
& +\eta^{i k} \eta^{00} \eta^{j m}\left(\partial_{i} x_{k}^{(2)}-\partial_{i} \partial_{k} x_{0}^{(1)}\right)\left(\partial_{j} x_{m}^{(2)}-\partial_{j} \partial_{m} x_{0}^{(1)}\right)+ \\
& +2 \eta^{i k} \eta^{00} \eta^{j m}\left(\partial_{k} v_{0}-v_{k}\right) \partial_{j} F_{i m}^{x_{1}}+ \\
& \left.\left.+\eta^{j l} \eta^{i m} \eta^{k n} \partial_{i} F_{l m}^{x_{1}} \partial_{k} F_{j n}^{x_{1}}\right)\right] .
\end{aligned}
$$

A new Lagrangian in an extended space can be constructed where Lagrange multipliers are introduced

$$
\begin{gathered}
\bar{L}^{v}=L^{v}+\pi_{(1)}^{k}\left(\partial_{0} x_{k}^{(1)}-x_{k}^{(2)}\right)+\pi_{(2)}^{k}\left(\partial_{0} x_{k}^{(2)}-v_{k}\right)+\pi_{(1)}^{0}\left(\partial_{0} x_{0}^{(1)}-v_{0}\right) \\
=\pi_{(1)}^{k} \partial_{0} x_{k}^{(1)}+\pi_{(2)}^{k} \partial_{0} x_{k}^{(2)}+\pi_{(1)}^{0} \partial_{0} x_{0}^{(1)}-H^{v}, \\
H^{v}=\pi_{(1)}^{k} x_{k}^{(2)}+\pi_{(2)}^{k} v_{k}+\pi_{(1)}^{0} v_{0}-L^{v},
\end{gathered}
$$

so that the configuration space now has "coordinates" $Z_{A}=\left\{x_{0}^{(1)}, x_{k}^{(1)}, x_{k}^{(2)}\right.$, $\left.v_{0}, v_{k}, \pi_{(1)}^{0}, \pi_{(1)}^{k}, \pi_{(2)}^{k}\right\}$, which are separated in the sets

$$
\begin{aligned}
t_{b} & =\left\{x_{0}^{(1)}, x_{k}^{(1)}, x_{k}^{(2)}, \pi_{(1)}^{0}, \pi_{(1)}^{k}, \pi_{(2)}^{k}\right\}, \\
t_{\beta} & =\left\{v_{0}, v_{k}\right\},
\end{aligned}
$$


with the following set of constraints

$$
\begin{array}{ccc}
\phi_{(1)}^{0}=p_{(1)}^{0}-\pi_{(1)}^{0}=0, & \phi_{(1)}^{k}=p_{(1)}^{k}-\pi_{(1)}^{k}=0, \\
\phi_{(2)}^{k}=p_{(2)}^{k}-\pi_{(2)}^{k}=0, & \phi_{v}^{0}=p_{v}^{0}=0, \\
\phi_{v}^{k}=p_{v}^{k}=0, & \phi_{0}^{\pi_{(1)}}=p_{0}^{\pi_{(1)}}=0, \\
\phi_{k}^{\pi_{(1)}}=p_{k}^{\pi_{(1)}}=0, & \phi_{k}^{\pi_{(2)}}=p_{k}^{\pi_{(2)}}=0, \\
\phi^{t}=p^{0}+H^{v}=0 .
\end{array}
$$

The integrability conditions must be evaluated. The first one gives

$$
\frac{\delta H^{v}(y)}{\delta v_{0}(x)}=0 \Rightarrow \pi_{(1)}^{0}=-2 a^{2} \eta^{00} \eta^{i k} \partial_{k}\left[\eta^{00}\left(\partial_{i} v_{0}-v_{i}\right)+\eta^{j m} \partial_{j} F_{i m}^{x_{1}}\right]
$$

The other conditions are

$$
\begin{gathered}
\pi_{(1)}^{k} \delta_{k}^{n} \delta(y-x)-\frac{\delta L^{v}(y)}{\delta v_{n}(x)}=0 \Rightarrow \\
\Rightarrow \pi_{(1)}^{n}=-2 a^{2} \eta^{00} \eta^{n i}\left[\eta^{00}\left(\partial_{i} v_{0}-v_{i}\right)+\eta^{j m} \partial_{j} F_{i m}^{x_{1}}\right] .
\end{gathered}
$$

At this point one observation must be made. From this last expression one can see that $v_{i}$ can be written as a function of $\pi_{(1)}^{n}, v_{0}$ and $F_{i m}^{x_{1}}$ :

$$
v_{i}=\frac{1}{2 a^{2}} \eta_{n i} \pi_{(1)}^{n}+\eta_{00} \eta^{j m} \partial_{j} F_{i m}^{x_{1}}+\partial_{i} v_{0}
$$

The same cannot be said about $v_{0}$, which remains undetermined. When one substitutes this result in $\pi_{(1)}^{0}$ it follows

$$
\pi_{(1)}^{0}=\partial_{k} \pi_{(1)}^{k} .
$$

The Generalized Brackets can be evaluated when the following brackets are calculated

$$
\begin{aligned}
\left\{\phi_{(1)}^{0}(x), \phi^{t}(y)\right\} & =-\int d z \frac{\delta H^{v}(y)}{\delta x_{0}^{(1)}(z)} \delta(z-x)= \\
& =\eta^{00} \eta^{i j}\left(x_{j}^{(2)}-\partial_{j} x_{0}^{(1)}\right) \partial_{i}^{y} \delta(y-x)+ \\
& -2 a^{2} \eta^{00} \eta^{i k} \eta^{j m}\left(\partial_{j} x_{m}^{(2)}-\partial_{j} \partial_{m} x_{0}^{(1)}\right) \partial_{i}^{y} \partial_{k}^{y} \delta(y-x) ; \\
\left\{\phi_{(1)}^{n}(x), \phi^{t}(y)\right\}= & -\eta^{i k} \eta^{j n} F_{i j}^{x_{1}} \partial_{k}^{y} \delta(y-x)+ \\
& -2 a^{2}\left[\eta^{n k} \eta^{j i}-\eta^{i k} \eta^{j n}\right] \eta^{00}\left(\partial_{k} v_{0}-v_{k}\right) \partial_{j}^{y} \partial_{i}^{y} \delta(y-x)+ \\
& -2 a^{2}\left[\eta^{j n} \eta^{i m}-\eta^{j m} \eta^{i n}\right] \eta^{k p} \partial_{k} F_{j p}^{x_{1}} \partial_{i}^{y} \partial_{m}^{y} \delta(y-x) ;
\end{aligned}
$$




$$
\begin{gathered}
\left\{\phi_{(2)}^{n}(x), \phi^{t}(y)\right\}=-\pi_{(1)}^{n} \delta(y-x)-\eta^{00} \eta^{i n}\left(x_{i}^{(2)}-\partial_{i} x_{0}^{(1)}\right) \delta(y-x)+ \\
+2 a^{2} \eta^{i k} \eta^{00} \eta^{j n}\left(\partial_{i} x_{k}^{(2)}-\partial_{i} \partial_{k} x_{0}^{(1)}\right) \partial_{j}^{y} \delta(y-x) ; \\
\left\{\phi_{0}^{\pi_{(1)}}(x), \phi^{t}(y)\right\}=-\frac{\delta H^{v}(y)}{\delta \pi_{(1)}^{0}(x)}=-v_{0} \delta(y-x) ; \\
\left\{\phi_{n}^{\pi_{(1)}}(x), \phi^{t}(y)\right\}=-\frac{\delta H^{v}(y)}{\delta \pi_{(1)}^{n}(x)}=-x_{n}^{(2)} \delta(y-x) ; \\
\left\{\phi_{n}^{\pi_{(2)}}(x), \phi^{t}(y)\right\}=-\frac{\delta H^{v}(y)}{\delta \pi_{(2)}^{n}(x)}=-v_{n} \delta(y-x) .
\end{gathered}
$$

The differential of any function $E$ on this extended space is

$$
\begin{aligned}
d E & =\frac{\partial E}{\partial t} d t+\left\{E, H^{v}\right\} d t+\left\{E, \phi_{v}^{0}\right\} d v_{0}+\left\{E, \phi_{v}^{k}\right\} d v_{k}+ \\
& +\int d w\left(\left\{E, \phi_{(1)}^{0}(w)\right\} v_{0}+\left\{E, \phi_{(1)}^{k}(w)\right\} x_{k}^{(2)}+\left\{E, \phi_{(2)}^{k}(w)\right\} v_{k}+\right. \\
& -\left\{E, \phi_{0}^{\pi_{(1)}}(w)\right\} \eta^{00} \eta^{i j} \partial_{i}^{w}\left(x_{j}^{(2)}-\partial_{j} x_{0}^{(1)}\right)+ \\
& -\left\{E, \phi_{0}^{\pi_{(1)}}(w)\right\} 2 a^{2} \eta^{00} \eta^{i k} \eta^{j m} \partial_{i}^{w} \partial_{k}^{w}\left(\partial_{j} x_{m}^{(2)}-\partial_{j} \partial_{m} x_{0}^{(1)}\right)+ \\
& +\left\{E, \phi_{n}^{\pi_{(1)}}(w)\right\} \eta^{i k} \eta^{j n} \partial_{k}^{w} F_{i j}^{x_{1}}+ \\
& -\left\{E, \phi_{n}^{\pi_{(1)}}(y)\right\} 2 a^{2}\left[\eta^{n k} \eta^{j i}-\eta^{i k} \eta^{j n}\right] \eta^{00} \partial_{j}^{w} \partial_{i}^{w}\left(\partial_{k} v_{0}-v_{k}\right)+ \\
& -\left\{E, \phi_{n}^{\pi_{(1)}}(w)\right\} 2 a^{2}\left[\eta^{j n} \eta^{i m}-\eta^{j m} \eta^{i n}\right] \eta^{k p} \partial_{i}^{w} \partial_{m}^{w} \partial_{k} F_{j p}^{x_{1}}+ \\
& -\left\{E, \phi_{n}^{\pi_{(2)}}(w)\right\} \pi_{(1)}^{n}-\left\{E, \phi_{n}^{\pi_{(2)}}(w)\right\} \eta^{00} \eta^{i n}\left(x_{i}^{(2)}-\partial_{i} x_{0}^{(1)}\right)+ \\
& \left.-\left\{E, \phi_{n}^{\pi_{(2)}}(w)\right\} 2 a^{2} \eta^{i k} \eta^{00} \eta^{j n} \partial_{j}^{w}\left(\partial_{i} x_{k}^{(2)}-\partial_{i} \partial_{k} x_{0}^{(1)}\right)\right) d t(w) .
\end{aligned}
$$

To obtain the equations of motion, it is necessary to consider $E=\left\{x_{0}^{(1)}\right.$, $\left.x_{k}^{(1)}, x_{k}^{(2)}, \pi_{(1)}^{0}, \pi_{(1)}^{k}, \pi_{(2)}^{k}\right\}$ :

$$
\begin{gathered}
d x_{0}^{(1)}=v_{0}(z) d t \\
d x_{p}^{(1)}=x_{p}^{(2)} d t \\
d x_{p}^{(2)}=v_{p} d t=\left[\frac{1}{2 a^{2}} \eta_{n p} \pi_{(1)}^{n}+\eta_{00} \eta^{j m} \partial_{j} F_{p m}^{x_{1}}+\partial_{p} v_{0}\right] d t \\
d \pi_{(1)}^{0}=-\left[\eta^{00} \eta^{i j} \partial_{i}\left(x_{j}^{(2)}-\partial_{j} x_{0}^{(1)}\right)+2 a^{2} \eta^{00} \eta^{i k} \eta^{j m} \partial_{i} \partial_{k}\left(\partial_{j} x_{m}^{(2)}-\partial_{j} \partial_{m} x_{0}^{(1)}\right)\right] d t \\
d \pi_{(1)}^{p}=\left(\eta^{i k} \eta^{j p} \partial_{k} F_{i j}^{x_{1}}+\eta_{00}\left[\eta^{i j} \partial_{i} \partial_{j} \pi_{(1)}^{p}-\eta^{i p} \partial_{i} \pi_{(1)}^{0}\right]\right) d t
\end{gathered}
$$




$$
\begin{aligned}
d \pi_{(2)}^{p} & =-\left(\pi_{(1)}^{p}+\eta^{00} \eta^{i p}\left(x_{i}^{(2)}-\partial_{i} x_{0}^{(1)}\right)+\right. \\
& \left.+2 a^{2} \eta^{i k} \eta^{00} \eta^{j p} \partial_{j}\left(\partial_{i} x_{k}^{(2)}-\partial_{i} \partial_{k} x_{0}^{(1)}\right)\right) d t .
\end{aligned}
$$

All the results obtained here are in accordance with those obtained in 22$]$ and [23], showing the consistency of the construction made in this work with the non first order HJ approach and with Dirac's procedure.

\subsection{Proca Model}

The next examples will be used to compare the two approaches introduced in this work.

Let us now consider the case of Proca Model, whose Lagrangian is

$$
\begin{gathered}
L=-\frac{1}{4} F_{\mu \nu} F^{\mu \nu}+\frac{f}{2} m^{2} A_{\mu} A^{\mu} \\
L=-\frac{1}{4}\left[2 \eta^{00} \eta^{i j}\left(\partial_{0} A_{i}-\partial_{i} A_{0}\right)\left(\partial_{0} A_{j}-\partial_{j} A_{0}\right)+\eta^{i k} \eta^{j n} F_{i j} F_{k n}\right]+ \\
+\eta^{00} \frac{f}{2} m^{2} A_{0} A_{0}+\eta^{i j} \frac{f}{2} m^{2} A_{i} A_{j}
\end{gathered}
$$

where $f= \pm 1$ according the convention of the metric, and $F_{\mu \nu}=\partial_{\mu} A_{\nu}-\partial_{\nu} A_{\mu}$.

\subsubsection{Standard approach}

Introducing new variables

$$
x_{\mu}^{(1)} \equiv A_{\mu}, \quad v_{\mu} \equiv \partial_{0} A_{\mu}=\partial_{0} x_{\mu}^{(1)},
$$

and defining

$$
F_{i j}^{x_{1}} \equiv \partial_{i} x_{j}^{(1)}-\partial_{j} x_{i}^{(1)},
$$

one finds

$$
\begin{aligned}
L^{v} & =-\frac{1}{4}\left[2 \eta^{00} \eta^{i j}\left(v_{i}-\partial_{i} x_{0}^{(1)}\right)\left(v_{j}-\partial_{j} x_{0}^{(1)}\right)+\eta^{i k} \eta^{j n} F_{i j}^{x_{1}} F_{k n}^{x_{1}}\right]+ \\
& +\eta^{\mu \nu} \frac{f}{2} m^{2} x_{\mu}^{(1)} x_{\nu}^{(1)} .
\end{aligned}
$$

Now one can define a new Lagrangian $\bar{L}^{v}$ in an extended space with "coordinates" $z_{A}=\left\{x_{\mu}^{(1)}, v_{\mu}, \pi^{\mu}\right\}$ such that

$$
\begin{gathered}
\bar{L}^{v}=L^{v}+\pi^{\mu}\left(\partial_{0} x_{\mu}^{(1)}-v_{\mu}\right)=\pi^{\mu} \partial_{0} x_{\mu}^{(1)}-H^{v}, \\
H^{v} \equiv \pi^{\mu} v_{\mu}-L^{v} .
\end{gathered}
$$


The constraints can be obtained:

$$
\begin{gathered}
\phi_{x}^{\mu}=p_{x}^{\mu}-\pi^{\mu}=0, \quad \phi_{v}^{\mu}=p_{v}^{\mu}=0, \\
\phi_{\mu}^{\pi}=p_{\mu}^{\pi}=0, \\
\phi^{t}=p^{0}+H^{v}=0 .
\end{gathered}
$$

The variables then are separated in two sets

$$
\begin{aligned}
t_{b} & =\left\{x_{\mu}^{(1)} ; \pi^{\mu}\right\}, \\
t_{\beta} & =\left\{v_{\mu}\right\},
\end{aligned}
$$

and one must obtain the integrability conditions:

$$
\begin{gathered}
\pi^{\mu} \delta_{\mu}^{\sigma} \delta(y-x)-\frac{\delta L^{v}(y)}{\delta v_{\sigma}(x)}=0, \\
\pi^{0}(x)=0 \\
\pi^{k}(x)=-\eta^{00} \eta^{i k}\left(v_{i}(x)-\partial_{i} x_{0}^{(1)}(x)\right)
\end{gathered}
$$

From this last expression it follows

$$
v_{i}=\partial_{i} x_{0}^{(1)}-\eta_{00} \eta_{k i} \pi^{k},
$$

while $v_{0}$ is not determined as a function of the other variables.

The total differential of any function $E=E\left(z_{A}\right)$ is given by

$$
\begin{aligned}
d E & =\left\{E, \phi^{t}\right\} d t+\left\{E, \phi_{v}^{\mu}\right\} d v_{\mu}+ \\
& -\int d z \int d x \int d w\left(\left\{E, \phi_{x}^{\nu}(z)\right\} \quad\left\{E, \phi_{\nu}^{\pi}(z)\right\}\right) \delta(z-x) . \\
& \cdot\left(\begin{array}{c}
-v_{\nu}(w) \delta(w-x) \\
\eta^{m n} \eta^{l \nu} F_{m l}^{x_{1}}(w) \partial_{n}^{w} \delta(w-x)-f m^{2} \eta^{\rho \nu} x_{\rho}^{(1)}(w) \delta(w-x)-C^{\nu}
\end{array}\right) d t(w),
\end{aligned}
$$

where

$$
C^{\sigma} \equiv \eta^{00} \eta^{n m}\left(v_{n}-\partial_{n} x_{0}^{(1)}\right) \delta_{0}^{\sigma} \partial_{m}^{w} \delta(w-x) .
$$

The equations of motion can be evaluated:

$$
\begin{gathered}
d x_{i}^{(1)}=\left(\partial_{i} x_{0}^{(1)}-\eta_{00} \eta_{k i} \pi^{k}\right) d t \\
d x_{0}^{(1)}=v_{0} d t \\
d \pi^{i}=\left[\eta^{m n} \eta^{l i} \partial_{n} F_{m l}^{x_{1}}+f m^{2} \eta^{m i} x_{m}^{(1)}\right] d t \\
d \pi^{0}=\left[f m^{2} \eta^{00} x_{0}^{(1)}-\eta^{00} \eta^{n m} \partial_{m}\left(v_{n}-\partial_{n} x_{0}^{(1)}\right)\right] d t
\end{gathered}
$$


With (38) and (37) it follows

$$
\begin{aligned}
d \pi^{0} & =\left[f m^{2} \eta^{00} x_{0}^{(1)}+\partial_{m} \pi^{m}\right] d t=0 \Rightarrow \\
& \Rightarrow x_{0}^{(1)}=-\frac{\eta_{00}}{f m^{2}} \partial_{m} \pi^{m}
\end{aligned}
$$

so that $x_{0}^{(1)}$ is written in terms of the $\pi^{m}$ and thereafter

$$
\begin{gathered}
v_{i}=-\eta_{00}\left(\frac{1}{f m^{2}} \partial_{i} \partial_{m}+\eta_{m i}\right) \pi^{m}, \\
d x_{i}^{(1)}=-\eta_{00}\left[\frac{1}{f m^{2}} \partial_{i} \partial_{m} \pi^{m}+\eta_{m i} \pi^{m}\right] d t ; \\
d \pi^{i}=\left[\eta^{m n} \eta^{l i} \partial_{n} F_{m l}^{x_{1}}+f m^{2} \eta^{m i} x_{m}^{(1)}\right] d t .
\end{gathered}
$$

\subsubsection{Degenerate Coordinates Approach}

The Lagrangian (33) shows that $A_{0}$ is a degenerate coordinate, i.e. $L=$ $L\left(A_{0}, A_{i}, \partial_{0} A_{i}\right)$, and $N_{A_{0}}=0, N_{A_{i}}=1$. Introducing new variables

$$
\begin{aligned}
v_{0} & \equiv A_{0}, \\
x_{i}^{(1)} & \equiv A_{i}, \quad v_{i} \equiv \partial_{0} A_{i}=\partial_{0} x_{i}^{(1)},
\end{aligned}
$$

and defining

$$
F_{i j}^{x_{1}} \equiv \partial_{i} x_{j}^{(1)}-\partial_{j} x_{i}^{(1)}
$$

it follows

$$
\begin{aligned}
L^{v} & =-\frac{1}{4}\left[2 \eta^{00} \eta^{i j}\left(v_{i}-\partial_{i} v_{0}\right)\left(v_{j}-\partial_{j} v_{0}\right)+\eta^{i k} \eta^{j n} F_{i j}^{x_{1}} F_{k n}^{x_{1}}\right]+ \\
& +\eta^{00} \frac{f}{2} m^{2} v_{0} v_{0}+\eta^{i j} \frac{f}{2} m^{2} x_{i}^{(1)} x_{j}^{(1)} .
\end{aligned}
$$

Now the new Lagrangian $\bar{L}^{v}$ with Lagrange multipliers can be written down:

$$
\begin{gathered}
\bar{L}^{v}=L^{v}+\pi^{i}\left(\partial_{0} x_{i}^{(1)}-v_{i}\right)=\pi^{i} \partial_{0} x_{i}^{(1)}-H^{v}, \\
H^{v} \equiv \pi^{i} v_{i}-L^{v} .
\end{gathered}
$$

The constraints are identified:

$$
\begin{gathered}
\phi_{x}^{i}=p_{x}^{i}-\pi^{i}=0, \quad \phi_{v}^{0}=p_{v}^{0}=0, \\
\phi_{v}^{i}=p_{v}^{i}=0, \quad \phi_{i}^{\pi}=p_{i}^{\pi}=0, \\
\phi^{t}=p^{0}+H^{v}=0,
\end{gathered}
$$


and the variables are separated:

$$
\begin{aligned}
t_{b} & =\left\{x_{i}^{(1)} ; \pi^{i}\right\}, \\
t_{\beta} & =\left\{v_{0} ; v_{i}\right\} .
\end{aligned}
$$

The integrability conditions must be determined:

$$
\begin{gathered}
\frac{\delta L^{v}(y)}{\delta v_{0}(x)}=\eta^{00} \eta^{i j}\left(v_{i}-\partial_{i} v_{0}\right) \partial_{j}^{y} \delta(y-x)+\eta^{00} f m^{2} v_{0} \delta(y-x)=0, \\
\pi^{k}(x)=-\eta^{00} \eta^{i k}\left(v_{i}(x)-\partial_{i} v_{0}(x)\right),
\end{gathered}
$$

from where it is immediate to verfiy that

$$
v_{i}=\partial_{i} v_{0}-\eta_{00} \eta_{k i} \pi^{k}
$$

Integrating (48), one finds

$$
v_{0}=-\frac{\eta_{00}}{f m^{2}} \partial_{j} \pi^{j}
$$

which leads one to conclude that

$$
v_{i}=-\eta_{00}\left(\frac{1}{f m^{2}} \partial_{i} \partial_{j}+\eta_{j i}\right) \pi^{j} .
$$

The differential of any function $E=E\left(z_{A}\right)$ is

$$
\begin{aligned}
d E & =\left\{E, \phi^{t}\right\} d t+\left\{E, \phi_{v}^{0}\right\} d v_{0}+\left\{E, \phi_{v}^{i}\right\} d v_{i}+ \\
& -\int d z \int d x \int d w\left(\left\{E, \phi_{x}^{j}(z)\right\} \quad\left\{E, \phi_{j}^{\pi}(z)\right\}\right) \delta(z-x) \\
& \cdot\left(\begin{array}{c}
-v_{j}(w) \delta(w-x) \\
\eta^{m n} \eta^{l j} F_{m l}^{x_{1}}(w) \partial_{n}^{w} \delta(w-x)-f m^{2} \eta^{m j} x_{m}^{(1)}(w) \delta(w-x)
\end{array}\right) d t(w),
\end{aligned}
$$

and the equations of motion are:

$$
\begin{gathered}
d x_{i}^{(1)}=-\eta_{00}\left(\frac{1}{f m^{2}} \partial_{i} \partial_{j}^{x}+\eta_{j i}\right) \pi^{j} d t \\
d \pi^{i}=\left[\eta^{m n} \eta^{l i} \partial_{n} F_{m l}^{x_{1}}+f m^{2} \eta^{m i} x_{m}^{(1)}\right] d t .
\end{gathered}
$$

It is interesting to notice that in the standard approach, the determination of $x_{0}^{(1)}$ (i.e. $\left.A_{0}\right)$ and $v_{i}$ does not occur when integrability conditions are evaluated, but it can only be obtained when equations of motion are considered and when a kind of consistency condition is applied $\left(\pi^{0}=0 \Rightarrow d \pi^{0}=0\right)$. In the degenerate approach, $v_{0}$ (i.e. $A_{0}$ ) and $v_{i}$ are readily determined by integrability conditions, no use of equations of motion are necessary and no extra condition must be applied. 


\section{$5.3 \quad$ QCD}

The Lagrangian of the gauge field of QCD is

$$
L=-\frac{1}{4} F_{\mu \nu}^{a} F_{a}^{\mu \nu},
$$

where $F_{\mu \nu}^{a}=\partial_{\mu} A_{\nu}^{a}-\partial_{\nu} A_{\mu}^{a}+f_{b c}^{a} A_{\mu}^{b} A_{\nu}^{c}$. Expliciting the time derivatives one finds:

$$
\begin{aligned}
L & =-\frac{1}{2} \eta^{00} \eta^{i j}\left(\partial_{0} A_{i}^{a}-\partial_{i} A_{0}^{a}+f_{b c}^{a} A_{0}^{b} A_{i}^{c}\right) \partial_{0} A_{a j}+ \\
& -\frac{1}{2} \eta^{00} \eta^{i j}\left(\partial_{0} A_{i}^{a}-\partial_{i} A_{0}^{a}+f_{b c}^{a} A_{0}^{b} A_{i}^{c}\right)\left(-\partial_{j} A_{a 0}+f_{d a e} A_{0}^{d} A_{j}^{e}\right)+F_{i j}^{a} F_{a}^{i j} .
\end{aligned}
$$

\subsubsection{Standard Approach}

With the new variables

$$
x_{\mu}^{a} \equiv A_{\mu}^{a}, \quad v_{\mu}^{a} \equiv \partial_{0} A_{\mu}^{a}=\partial_{0} x_{\mu}^{a},
$$

and with the definition

$$
F_{i j}^{a(x)} \equiv \partial_{i} x_{j}^{a}-\partial_{j} x_{i}^{a}+f_{d e}^{a} x_{i}^{d} x_{j}^{e},
$$

it follows

$$
\begin{gathered}
L^{v}=-\frac{1}{4}\left[2 \eta^{00} \eta^{i j}\left(v_{i}^{a}-\partial_{i} x_{0}^{a}+f_{b c}^{a} x_{0}^{b} x_{i}^{c}\right) v_{a j}+\right. \\
\left.+2 \eta^{00} \eta^{i j}\left(v_{i}^{a}-\partial_{i} x_{0}^{a}+f_{b c}^{a} x_{0}^{b} x_{i}^{c}\right)\left(-\partial_{j} x_{a 0}+f_{d a e} x_{0}^{d} x_{j}^{e}\right)+F_{i j}^{a(x)} F_{a(x)}^{i j}\right] \\
\bar{L}^{v}=\pi_{a}^{\mu} \partial_{0} x_{\mu}^{a}-H^{v} \\
H^{v} \equiv \pi_{a}^{\mu} v_{\mu}^{a}-L^{v}
\end{gathered}
$$

The constraints are identified

$$
\begin{gathered}
\phi_{(x) a}^{\mu}=p_{(x) a}^{\mu}-\pi_{a}^{\mu}=0, \quad \phi_{(v) a}^{\mu}=p_{(v) a}^{\mu}=0, \\
\phi_{\mu}^{(\pi) a}=p_{\mu}^{(\pi) a}=0, \\
\phi^{t}=p^{0}+H^{v}=0,
\end{gathered}
$$

and the variables are separated

$$
\begin{aligned}
t_{b} & =\left\{x_{\mu}^{a} ; \pi_{a}^{\mu}\right\}, \\
t_{\beta} & =\left\{v_{\mu}^{a}\right\} .
\end{aligned}
$$

The conditions that fix the subspace are

$$
\pi_{c}^{0} \delta(y-x)=\frac{\delta L^{v}(y)}{\delta v_{0}^{c}(x)}=0,
$$




$$
\pi_{c}^{k}(x)=-\eta^{00} \eta^{i k}\left(v_{c i}-\partial_{i} x_{c 0}+f_{b c d} x_{0}^{b} x_{i}^{d}\right),
$$

from where one obtains

$$
v_{c i}=\partial_{i} x_{c 0}-f_{b c d} x_{0}^{b} x_{i}^{d}-\eta_{00} \eta_{i k} \pi_{c}^{k},
$$

while $v_{0}^{c}$ remains undetermined.

$d E$ is given by

$$
\begin{aligned}
d E & =\left\{E, \phi^{t}\right\} d t+\left\{E, \phi_{(v) a}^{\mu}\right\} d v_{\mu}^{a}+ \\
& -\int d z \int d y \int d w\left(\left\{E, \phi_{(x) a}^{\mu}(z)\right\} \quad\left\{E, \phi_{\mu}^{(\pi) a}(z)\right\}\right) . \\
& \cdot\left(\begin{array}{c}
-v_{\mu}^{a}(w) \delta(w-y) \\
-\left(C_{a}^{\mu}(w-y)+B_{a}^{\mu}(w-y)\right)
\end{array}\right) \delta(z-y) d t(w),
\end{aligned}
$$

where

$$
\begin{aligned}
C_{a}^{\mu} & \equiv-\eta^{00} \eta^{i j}\left(v_{e i}-\partial_{i}^{w} x_{e 0}+f_{d e f} x_{0}^{d} x_{i}^{f}\right) . \\
& \cdot\left(-\delta_{a}^{e} \delta_{0}^{\mu} \partial_{j}^{w} \delta(w-y)+f_{a c}^{e} \delta_{0}^{\mu} \delta(w-y) x_{j}^{c}+f_{b a}^{e} x_{0}^{b} \delta_{j}^{\mu} \delta(w-y)\right), \\
B_{a}^{\mu} & \equiv-\frac{1}{2} F_{e(x)}^{i j}\left(\delta_{a}^{e} \delta_{j}^{\mu} \partial_{i}^{w} \delta(w-y)-\delta_{a}^{e} \delta_{i}^{\mu} \partial_{j}^{w} \delta(w-y)+\right. \\
& \left.+f_{a{ }_{c}{ }^{e} \delta_{i}^{\mu}}^{\mu} \delta(w-y) x_{j}^{c}+f_{d a}^{e} x_{i}^{d} \delta_{j}^{\mu} \delta(w-y)\right) .
\end{aligned}
$$

The equations of motion are obtained:

$$
\begin{gathered}
d x_{\rho}^{g}(u)=v_{\rho}^{g}(u) d t \\
d x_{i}^{g}=\left(\partial_{i} x_{0}^{g}-f_{b}^{g}{ }_{d}^{b} x_{0}^{b} x_{i}^{d}-\eta_{00} \eta_{i k} \pi^{g k}\right) d t \\
d x_{0}^{g}=v_{0}^{g} d t \\
d \pi_{a}^{\mu}(u)=-\eta^{00} \eta^{i j}\left[\delta_{0}^{\mu} D_{j a}^{e}\left(v_{e i}-\partial_{i} x_{e 0}+f_{d e f} x_{0}^{d} x_{i}^{f}\right)+\right. \\
\left.+\delta_{j}^{\mu} f_{b a}^{e} x_{0}^{b}\left(v_{e i}-\partial_{i} x_{e 0}+f_{d e f} x_{0}^{d} x_{i}^{f}\right)\right](u) d t(u)+ \\
+\delta_{j}^{\mu} D_{i a}^{e} F_{e(x)}^{i j}(u) d t(u),
\end{gathered}
$$

where

$$
D_{i a}^{e} F_{e(x)}^{i j}=\delta_{a}^{e} \partial_{i} F_{e(x)}^{i j}+f_{a c}^{e} x_{i}^{c} F_{e(x)}^{i j},
$$

which leads to

$$
\begin{gathered}
d \pi_{a}^{0}=-\eta^{00} \eta^{i j} D_{j a}^{e}\left(v_{e i}-\partial_{i}^{w} x_{e 0}+f_{d e f} x_{0}^{d} x_{i}^{f}\right) d t \\
d \pi_{a}^{k}(u)=-\eta^{00} \eta^{i k} f_{b a}^{e} x_{0}^{b}\left(v_{e i}-\partial_{i}^{w} x_{e 0}+f_{d e f} x_{0}^{d} x_{i}^{f}\right)(u) d t+D_{i a}^{e} F_{e(x)}^{i k} d t .
\end{gathered}
$$


With conditions (61) and (60) it follows

$$
\begin{gathered}
d \pi_{a}^{k}(u)=f_{b a}^{e} x_{0}^{b} \pi_{e}^{k} d t+D_{i a}^{e} F_{e(x)}^{i k} d t, \\
d \pi_{a}^{0}=D_{j a}^{e} \pi_{e}^{j} d t=0 \Rightarrow D_{j a}^{e} \pi_{e}^{j}=0 .
\end{gathered}
$$

This last result is the non-abelian generalization of the Gauss law and it arises only when one considers $d \pi_{a}^{0}=0$.

\subsubsection{Degenerate Coordinates Approach}

Since $L=L\left(A_{0}^{a}, A_{i}^{a}, \partial_{0} A_{i}^{a}\right)$, i.e. $N_{A_{0}^{a}}=0, N_{A_{i}^{a}}=1$, the new variables will be

$$
\begin{aligned}
v_{0}^{a} & \equiv A_{0}^{a}, \\
x_{i}^{a} & \equiv A_{i}^{a}, \quad v_{i}^{a} \equiv \partial_{0} A_{i}^{a}=\partial_{0} x_{i}^{a} .
\end{aligned}
$$

With

$$
F_{i j}^{a(x)} \equiv \partial_{i} x_{j}^{a}-\partial_{j} x_{i}^{a}+f_{d a e} x_{i}^{d} x_{j}^{e},
$$

it follows

$$
\begin{gathered}
L^{v}=-\frac{1}{4}\left[2 \eta^{00} \eta^{i j} v_{i}^{a} v_{a j}+4 \eta^{00} \eta^{i j} v_{i}^{a}\left(-\partial_{j} v_{a 0}+f_{d a e} v_{0}^{d} x_{j}^{e}\right)+\right. \\
\left.+2 \eta^{00} \eta^{i j}\left[-\partial_{i} v_{0}^{a}+f_{b c}^{a} v_{0}^{b} x_{i}^{c}\right]\left[-\partial_{j} v_{a 0}+f_{d a e} v_{0}^{d} x_{j}^{e}\right]+F_{i j}^{a(x)} F_{a(x)}^{i j}\right], \\
\bar{L}^{v}=\pi_{a}^{i} \partial_{0} x_{i}^{a}-H^{v}, \\
H^{v} \equiv \pi_{a}^{i} v_{i}^{a}-L^{v},
\end{gathered}
$$

and

$$
\begin{gathered}
\phi_{a(x)}^{i}=p_{a(x)}^{i}-\pi_{a}^{i}=0, \quad \phi_{a(v)}^{0}=p_{a(v)}^{0}=0, \\
\phi_{a(v)}^{i}=p_{a(v)}^{i}=0, \quad \phi_{i}^{a(\pi)}=p_{i}^{a(\pi)}=0, \\
\phi^{t}=p^{0}+H^{v}=0 .
\end{gathered}
$$

One has

$$
\begin{aligned}
t_{b_{x}} & =\left\{x_{i}^{a} ; \pi_{a}^{i}\right\}, \\
t_{\beta_{x}} & =\left\{v_{0}^{a} ; v_{i}^{a}\right\} .
\end{aligned}
$$

The integrability conditions are

$$
\begin{gathered}
\eta^{00} \eta^{i j} \partial_{j} \partial_{i} v_{g 0}-\eta^{00} \eta^{i j} \partial_{j} v_{g i}-\eta^{00} \eta^{i j} f_{g a e} v_{i}^{a} x_{j}^{e}+\eta^{00} \eta^{i j} f_{g a c} x_{j}^{c} \partial_{i} v_{0}^{a}+ \\
-\eta^{00} \eta^{i j} f_{a g c} x_{j}^{c} \partial_{i} v_{0}^{a}-\eta^{00} \eta^{i j} f_{a g c} v_{0}^{a} \partial_{i} x_{j}^{c}-\eta^{00} \eta^{i j} f_{g c}^{a} f_{b a e} v_{0}^{b} x_{j}^{e} x_{i}^{c}=0, \\
\pi_{g}^{k}(x)=-\eta^{00} v_{g}^{k}+\eta^{00} \partial^{k} v_{g 0}-\eta^{00} f_{d g e} v_{0}^{d} x^{e k}
\end{gathered}
$$


From this last result one finds

$$
v_{g}^{k}=-\eta_{00} \pi_{g}^{k}+\partial^{k} v_{g 0}-f_{d g e} v_{0}^{d} x^{e k},
$$

which, with the previous condition, leads to

$$
\eta^{i j} D_{j g}^{e} \pi_{e i}=\eta^{i j} \partial_{j} \pi_{g i}+\eta^{i j} f_{g e}^{a} x_{j}^{e} \pi_{a i}=0 .
$$

$d E$ can be constructed,

$$
\begin{aligned}
d E & =\left\{E, \phi^{t}\right\} d t+\left\{E, \phi_{g(v)}^{0}\right\} d v_{0}^{g}+\left\{E, \phi_{g(v)}^{i}\right\} d v_{i}^{g}+ \\
& -\int d x \int d w\left(\left\{E, \phi_{g(x)}^{i}(x)\right\} \quad\left\{E, \phi_{i}^{g(\pi)}(x)\right\}\right) . \\
& \cdot\left(\begin{array}{c}
-v_{i}^{g}(w) \delta(w-x) \\
-\frac{\delta L^{v}(w)}{\delta x_{i}^{g}(x)}
\end{array}\right) d t(w),
\end{aligned}
$$

and the equations of motion are stablished:

$$
\begin{aligned}
d x_{g}^{k} & =\left(-\eta_{00} \pi_{g}^{k}+\partial^{k} v_{g 0}-f_{d g e} v_{0}^{d} x^{e k}\right) d t, \\
d \pi_{g}^{i}(y) & =\left[\partial_{k} F_{g(x)}^{k i}-F_{a(x)}^{i k} f_{g}^{a} x^{b} x_{k}^{b}+\right. \\
& -\eta^{00} \eta^{k i} f_{d a g} v_{k}^{a} v_{0}^{d}+\eta^{00} \eta^{k i} f_{b a g} v_{0}^{b} \partial_{k} v_{0}^{a}+ \\
& \left.-\eta^{00} \eta^{i j} f_{b g}^{a} f_{d a c} v_{0}^{b} v_{0}^{d} x_{j}^{c}\right] d t(w) .
\end{aligned}
$$

With (72) it follows

$$
d \pi_{g}^{i}=\left(D_{k g}^{e} F_{e(x)}^{k i}+f_{b g}^{a} v_{0}^{b} \pi_{a}^{i}\right) d t .
$$

One can note that, for QCD in both approaches, $v_{g}^{k}$ have been determined by integrability conditions. In the degenerate approach, the non-abelian generalization of the Gauss law, $D_{j a}^{e} \pi_{e}^{j}=0$, arises as an integrability condition, while in the standard one it can be obtained only when equations of motion and a consistency condition $\left(\pi_{a}^{0}=0 \Rightarrow d \pi_{a}^{0}=0\right)$ are applied.

\section{Final Remarks}

In this work we could see how the first order Hamilton-Jacobi approach can be used to describe systems with higher order derivatives. With an extension of the configuration space, we were able to make two different approaches for such systems in analogy to what is known in the hamiltonian formalism (SA and DC). As it is seen in this work, in the HJ context we see that all results obtained in the SA are obtained in the DC description. However it is important to notice that it was possible only when a kind of consistency condition was used 
in the SA. This seems to be an advantage of the DC approach, where no extra condition must be considered and therefore less calculations must be made.

One interesting feature of the application of the first order HJ formalism to DC approach is that no redefinition of the singularity of the theory had to be made, as it happens in [13. According to Gitman and Tyutin, the gauge character of the theory would strictly correlated to the singularity of the new Hessian matrix proposed by them when degenerate coordinates exist. This is an analysis that cannot be made in this work, since no redefinition of the Hessian matrix has been done here. However, what one can see in the example of the QCD (which is a gauge theory with degenerate coordinates) is that, in the HJ first order approach, perhaps this redefinition is not necessary.

\section{Acknowledgements}

BMP would like to thank D. M. Gitman for providing the reference [11] and BMP and PJP would like to thank D. M. Gitman for introducing them to the reference [13] and the discussions about it.

BMP was partially supported by CNPq and FAPESP; MCB was supported by CAPES; PJP would like to thank CTA staff for incentive.

\section{References}

[1] M. Ostrogradski, Mem. Ac. St. Petersbourg 1 (1850) 385 .

[2] B. Podolsky, Phys. Rev. 62 (1942) 68; B. Podolsky and C. Kikuchi, Phys. Rev. 65 (1944) 228; B. Podolsky and P. Schwed, Rev. Mod. Phys. 20 (1948) 40 .

[3] F. Bopp, Ann. Physik 38 (1940) 345.

[4] A. Green, Phys. Rev. 73 (1948) 26; 75 (1949) 1926.

[5] K. S. Stelle, Phys. Rev. D 16 (1977) 953.

[6] P. West, Nucl. Phys. B. 268 (1986) 113.

[7] A. I. Alekseev, B. A. Arbuzov and V. A. Baikov, Theor. Math. Phys. 52 (1982) $739 ; 59$ (1983) 372.

[8] R. R. Cuzinatto, C. A. M. de Melo and P. J. Pompeia, Annals of Physics, doi:10.10.16/j.aop.2006.07.006; R. R. Cuzinatto, C. A. M. de Melo and P. J. Pompeia, Gauge Formulation for Higher Order Gravity, gr-qc/0611116.

[9] P. A. M. Dirac, Can. J. Math. 2 (1950) 129; 3 (1951) 129.

[10] P. A. M. Dirac, Lectures on Quantum Mechanics (Belfer Graduate School of Science, Yeshiva University, New York, 1964).

[11] D. M. Gitman, S. L. Lyakhovich and I. V. Tyutin, Soviet Phys. Journ. 26 (1983) 730. 
[12] D. M. Gitman and I. V. Tyutin, Quantization of Fields with Constraints (Springer-Verlag, New York/ Berlin, 1990).

[13] D. M. Gitman and I. V. Tyutin, Nucl. Phys. B 630 (2002) 509.

[14] C. Carathéodory, Calculus of Variations and Partial Differential Equations of the First Order (AMS Chelsea Publishing Company, 1999).

[15] Y. Güler, Il Nuovo Cimento B 107 (1992) 1398; 107 (1992) 1143.

[16] B. M. Pimentel, R. G. Teixeira and J. L. Tomazelli, Ann. Phys. 267 (1998) 75.

[17] Y. Güler, Il Nuovo Cimento B 109 (1994) 341; 111 (1996) 513.

[18] Y. Güler and D. Baleanu, Il Nuovo Cimento B 114 (1999) 1023; 115 (2000) 319.

[19] B. M. Pimentel, P. J. Pompeia, J. F. da Rocha-Neto and R. G. Teixeira, Gen. Rel. Grav. 35 (2003) 877.

[20] B. M. Pimentel, P. J. Pompeia, J. F. da Rocha-Neto, Il Nuovo Cimento B $120(2005) 981$.

[21] Soon-Tae Hong, Yong-Wan Kim, Young-Jai Park, K. D. Rothe, Mod. Phys. Lett. A 17 (2002) 435.

[22] B. M. Pimentel and R. G. Teixeira, Il Nuovo Cimento B 111 (1996) 841.

[23] C. A. P. Galvão and B. M. Pimentel, Can. J. Phys. 66 (1988) 460.

[24] B. M. Pimentel and R. G. Teixeira, Il Nuovo Cimento B 113 (1998) 805.

[25] M. C. Bertin, B. M. Pimentel and P. J. Pompeia, Mod. Phys. Lett. A 20 (2005) 2873. 